\title{
Moving beyond the Solvent-tip Approximation to Determine Site-specific Variations of Interfacial Water Structure through 3D Force Microscopy
}

Elias Nakouzi, ${ }^{1}$ Andrew G. Stack, ${ }^{2}$ Sebastien Kerisit, ${ }^{1}$ Benjamin A. Legg, ${ }^{1,6}$ Christopher J. Mundy, ${ }^{1,3}$ Gregory K. Schenter, ${ }^{1,4^{*}}$ Jaehun Chun, ${ }^{1,5^{*}}$ and James J. De Yoreo ${ }^{1,6^{*}}$

1 Physical and Computational Sciences Directorate, Pacific Northwest National Laboratory, Richland, WA 99354, USA.

${ }^{2}$ Chemical Sciences Division, Oak Ridge National Laboratory, 1 Bethel Valley Rd., Oak Ridge, TN 37830, USA.

${ }^{3}$ Department of Chemical Engineering, University of Washington, Seattle, WA 98195, USA.

${ }^{4}$ Department of Chemistry, Washington State University, Pullman, WA 99164, USA.

${ }^{5}$ Benjamin Levich Institute, CUNY City College of New York, New York, NY 10031, USA.

${ }^{6}$ Department of Materials Science and Engineering, University of Washington, Seattle, WA 98195, USA. 


\section{Supplementary Information}

\section{Acquiring and characterizing geological boehmite crystals}

The rocks acquired from the Saga quarries in Tvedalen (Norway) consisted mostly of a natrolite matrix (Figure S1a). Embedded millimeter-sized boehmite crystals (Figure S1b), with large flat terraces (Figure S1c), were retrieved using tweezers. The boehmite single crystals were analyzed using X-ray diffraction (Figure S2a) and a Horiba Raman spectrometer (Figure S2b). After chemical and crystallographic identification, a large boehmite single crystal was mounted and glued to an AFM stub (Ted Pella Inc) using Crystalbond 555 (Ted Pella Inc). The crystal is cleaved to expose a fresh (010) plane for AFM measurements.

\section{AFM measurements}

As mentioned in the main manuscript text, the AFM measurements were performed using an Asylum Research Cypher Video Rate Scanning (VRS) atomic force microscope operated in amplitude modulated mode. The cantilever was driven photothermally at its resonance frequency. The drive amplitude in bulk solution was set at $<0.1 \mathrm{~nm}$ and the phase shift was centered at 90 degrees. The nominal tip radius provided by the manufacturer tips is $5-10 \mathrm{~nm}$, but TEM imaging revealed a smaller radius of $\sim 1.5 \mathrm{~nm}$ (Figure 5a).

To process the data, we first correct for the slope of the imaged surface by subtracting the average height for each row or linescan from the data points in this line. The sinusoidal trajectory of the tip for each individual force curve is binned and linearized to obtain a unified vector for the tip height $z$. To reduce the effect of measurement noise, the data is passed through a 3D median filter. Subsequently, the data set is sheared to transform the translational symmetry vectors measured by Fourier transform into the expected orthorhombic symmetry known for the boehmite(010) plane. Note that a detailed manuscript on the protocol of acquiring, processing, and analyzing 3D fast force mapping is currently in preparation. 


\section{Boehmite-water molecular dynamics simulations}

The MD simulations of the boehmite-water-silica system were performed using DL_POLY Classic (1). Atoms are represented as point-charge particles that interact through longrange Coulombic forces and short-range interactions, whereby the latter were described by parameterized functions and represented the repulsion between electron-charge clouds, van der Waals attraction forces, and many-body terms, such as bond angle bending. (Details of the resulting bond lengths are given in the SI section on the boehmitewater LJ object simulations). The potential parameters were those of the CLAYFF force field (2), which uses the flexible SPC model (3) to represent water. The MD simulations were performed at $300 \mathrm{~K}$ and zero applied pressure in the NVT ensemble (constant number of particles, constant volume, and constant temperature). The temperature was kept constant using the Nosé-Hoover thermostat (4). The electrostatic interactions were calculated using the Ewald summation method (5). A 10 A cutoff was used for the shortrange interactions and the real part of the Ewald sum. The Verlet leapfrog algorithm was used to integrate the equation of motion with a $1 \mathrm{fs}$ time step.

\section{Boehmite-water-silica simulations}

Amorphous silica was generated by first melting a $8 \times 8 \times 8 \alpha$-quartz supercell at $6000 \mathrm{~K}$ for $200 \mathrm{ps}$ in the NVT ensemble and then quenching it to $300 \mathrm{~K}$ at a rate of $5 \mathrm{~K} / \mathrm{ps}$, followed by two subsequent 100-ps simulations in the NPT and NVE ensembles. A nanoparticle with a radius of $10 \AA$ was excised from the resulting bulk amorphous silica structure. Protons and hydroxyl/aquo groups were then added to undercoordinated oxygen and silicon atoms with the proportion of hydroxyl and aquo groups set to achieve a chargeneutral nanoparticle.

The boehmite-water simulation cell contained a boehmite slab $20 \AA$ in thickness with a surface area of $67.08 \times 64.93 \AA^{2}$ and a water slab approximately $65 \AA$ in thickness containing 8857 water molecules. The number of water molecules was selected to ensure a water density of $1 \mathrm{~g} \mathrm{~cm}^{-3}$ away from the mineral substrate. A MD simulation of the 
boehmite-water interface without the nanoparticle was first run for 2 ns to compute the interfacial water structure.

Potential of mean force (PMF) calculations were performed to determine the free energy of the amorphous silica nanoparticle interacting with the semi-infinite boehmite substrate in water as a function of the distance $h$ along the normal to the surface ( $z$-direction). The free energy difference between the particle at distance $h$ and the particle far away from the substrate (at distance $h_{0}$ ) was calculated by integrating the ensemble average of the normal force on the whole nanoparticle, $\left\langle f_{z}>\right.$, over the distance from $h_{0}$ to $h$

$$
\Delta A(h)=\int_{\infty}^{h}\left\langle f_{z}(h)\right\rangle \mathrm{d} h \approx \int_{h_{0}}^{h}\left\langle f_{z}(h)\right\rangle \mathrm{d} h
$$

where $h$ was varied with $0.25 \AA$ intervals from 10.7 to $25.7 \AA$. For each value of $h$, the nanoparticle was added to the boehmite-water system with its COM at the appropriate distance and all water molecules with their oxygen atoms within $2.3 \AA$ of any atom of the nanoparticle removed. An energy minimization followed by a 500 ps MD simulation with the nanoparticle and boehmite substrate frozen (with the velocities scaled to $300 \mathrm{~K}$ for the first $100 \mathrm{ps}$ ) were first performed to relax the water structure around the nanoparticle. A 500 ps PMF calculation (with the velocities scaled to $300 \mathrm{~K}$ for the first $50 \mathrm{ps}$ ) was then performed to collect the force data. The middle Al layer of the boehmite substrate was kept frozen during the latter calculation to ensure that the substrate would not move the reduce the total force on the nanoparticle.

In each PMF calculation and following the approach described by Zhang et al. (6) and Kerisit and De Yoreo (7), the overall rotation about the center of mass (COM) of the nanoparticle about all three axes was removed. The nanoparticle was free to translate in the $x-y$ plane. At the start of each individual PMF calculation, the initial atomic velocities were modified to remove the initial angular momentum and COM motion of the nanoparticle. Then, at each MD step, the net torque on the nanoparticle about the three axes and the net force on the nanoparticle along the $z$ axis were removed after the force calculation, that is, before the integration of the equations of motion. In addition, because the atomic velocities are automatically scaled to the target temperature during the 
equilibration period, the nanoparticle's angular momentum and COM motion were also removed at each MD step during equilibration.

\section{Analysis of overlap between boehmite and silica hydration structure}

Using the assumption in Equation 4, the solvation-mediated energy is given by (8):

$$
W\left(\boldsymbol{R}_{1}, \boldsymbol{R}_{2}\right)=-k T \ln \left[1+\sum_{i} \rho_{i} \int\left(\frac{\rho_{i}\left(\boldsymbol{R} \mid \boldsymbol{R}_{\mathbf{1}}\right)}{\rho_{i}^{o}}-1\right)\left(\frac{\rho_{i}\left(\boldsymbol{R} \mid \boldsymbol{R}_{2}\right)}{\rho_{i}^{o}}-1\right) d \boldsymbol{R}\right]
$$

where $i$ denotes the different species in bulk solution; in this case, we consider only one species, namely water molecules, $\rho$ and $\rho$ o correspond to the local and bulk water densities, respectively. An additional assumption is made to convert the density of the silanol groups at the surface of the silica particle to equivalent water density due to their propensity for hydrogen bonding. Since the silanol groups are more localized than water molecules, we use a conversion rate of 3:1. However, the results presented in Figure 6 are robust to the choice of this ratio. 


\section{Boehmite-Water-LJ Object Simulations}

A supercell was simulated consisting of $6 \times 8 \times 3$ boehmite unit cells with dimensions $22.639 \times 23.406 \times 37.355 \AA^{3}$ in the [001], [100], and [010] directions. These simulations were also performed using CLAYFF forcefield ${ }^{2}$ (Table S1) and the lammps simulation package (9). Equilibrating in the isothermal-isobaric (NPT) ensemble yielded a cell volume $\sim 5.3 \%$ smaller than an experimental measure (10), in line with the force field as originally implemented. The average bond length of Al-Obridging is $1.933 \AA$, which compares well to the experimental bond length of $1.918 \AA$. The Al-Ohydroxyl, bond length is $1.895 \AA$, whereas the experimental measure is $1.902 \AA$. OH bond lengths are $1.049 \AA$, which compares to $0.928 \AA$ experimental value. From here, the dimensions of the supercell were doubled in the [010] direction (i.e., surface normal) and water was added during canonical ensemble (NVT) simulations until the equilibrated bulk-like water density was 0.101 atoms $/ \AA^{3}$, close to the actual value of 0.100 atoms $/ \AA^{3}$. The water force-field used was $\mathrm{F} 3 \mathrm{C}$, with a scaling parameter set to 1 (11). Cross-terms were implemented using geometric (D0) and arithmetic means (R0). A particle-particle particle-mesh solver was used for long range coulombic forces, and short range force were calculated at less than or equal to $10.0 \AA$. Interaction potentials were shifted to zero at $10 \AA$. The time step was $1 \mathrm{fs}$.

The relaxation constant of the Nosé-Hoover thermostat was set to $100 \mathrm{fs}$ and 500 fs in the LJ-object and PMF simulations, respectively. In both sets of simulations, zero pressure was achieved by running a periodic, bulk boehmite simulation in the NPT ensemble to allow the supercell to relax to an equilibrium state. Then, the supercell was expanded to accommodate a periodic box of water of equal dimensions as the boehmite slab, and water molecules were added until the bulk-like density of water in a NVT simulation of the interface was $0.033 \mathrm{H}_{2} \mathrm{O}$ molecules/ $\AA^{3}$.

Moreover, convergence was monitored by plotting the average free energy as a function of $z$ distance as the number of hills was increased. Simulations were re-run until the free energy minima at $\sim 0.2 \mathrm{~nm}$ and local maximum at $\sim 0.4 \mathrm{~nm}$ had converged. Distances further from the surface from that, e.g., $>0.4 \mathrm{~nm}$ are less well-converged, but these are also less important to interpreting the AM-AFM data. 
Table S1: Pair potential parameters used for boehmite-water-LJ object simulations.

\begin{tabular}{|c|c|c|c|}
\hline Atom type & Charge (e) & $\mathrm{D}_{\text {o }}(\AA \mathbf{A})$ & $R_{\circ}(\AA)$ \\
\hline $\mathrm{Al}$ & 1.575 & $1.3298 \mathrm{e}-6$ & 4.7943 \\
\hline Obridging & -1.05 & 0.1554 & 3.5532 \\
\hline Ohydroxyl & -0.95 & 0.1554 & 3.5532 \\
\hline $\mathrm{H}_{\text {hydroxyl }}$ & 0.425 & 0.0 & 0.0 \\
\hline $\mathrm{O}_{\text {water }}$ & -0.820 & 0.1848 & 3.5532 \\
\hline $\mathrm{H}_{\text {water }}$ & 0.410 & 0.0100 & 0.900 \\
\hline LJ Object & 0.000 & 0.2000 & 5.000 \\
\hline
\end{tabular}

To simulate the LJ object, a seventh atom type was created with zero charge, the approximate size of an aluminum atom, but with a potential well depth comparable to an oxygen on water. To derive the free energy of interaction of this object with the interface, the position of the object in the $\mathrm{x}, \mathrm{y}$ and $\mathrm{z}$ directions was biased using well-tempered metadynamics (12) (bias factor 20) through the plumed plugin (13). The multiple walkers method (14) was employed to more efficiently sample free energy space. To eliminate error due to translation of the supercell, eight aluminum atoms within the interior of the cell were frozen, i.e., left out of the MD loop. Gaussian hills of height $0.1 \mathrm{kcal} / \mathrm{mol}$ and width $0.1 \AA$ were added every 250 time steps for a total of $\sim 1.5$ million hills added in each $x, y$ and $z$ directions. To reduce simulation time, a wall potential was used to restrict travel of the LJ object to within $1 \mathrm{~nm}$ of the boehmite surface.

A histogram of the free energy of interaction of the LJ object with the interface was created by summing the Hills at a bin size of $0.4 \AA$. To mimic the force of interaction of a tip impinging on a surface, the derivative of the free energy was taken with respect to the surface normal to obtain a force in $\mathrm{pN}$. The force histogram was then interrogated to obtain site-specific forces shown in the main text (Figure 6b). 



\section{Supplementary Figures}
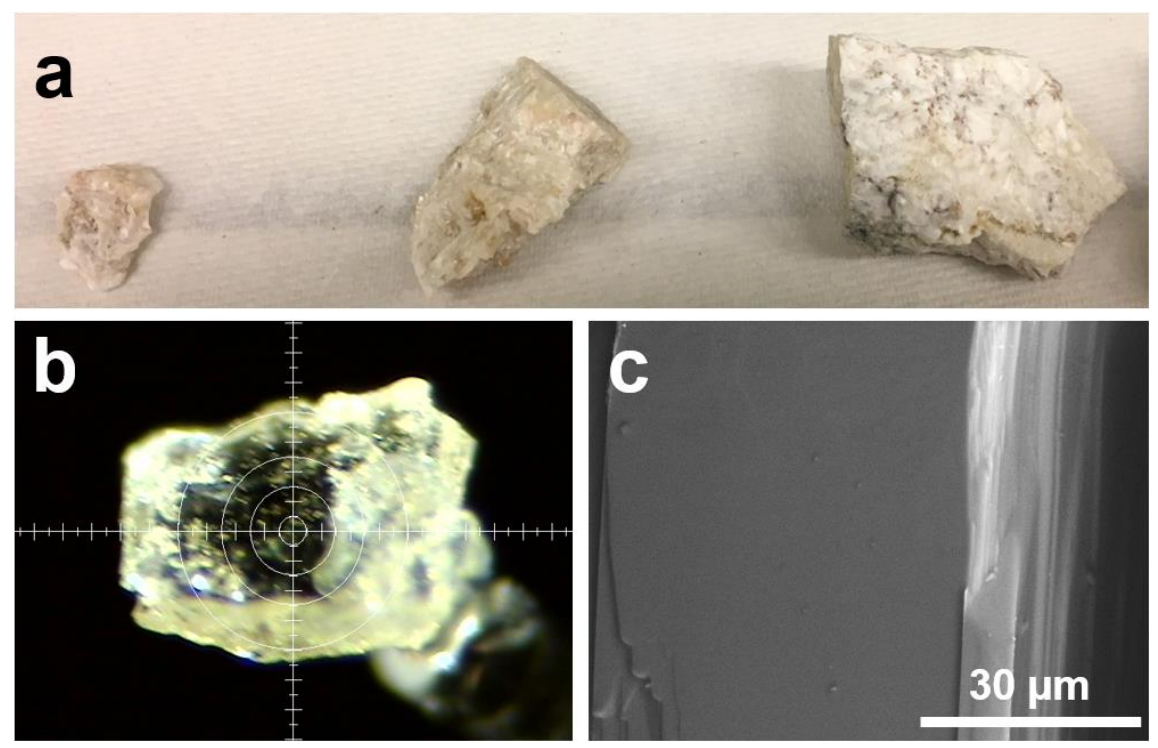

Figure S1. (a) Natrolite rocks with (b) embedded boehmite crystals. (c) SEM image shows large terraces of the boehmite surface.
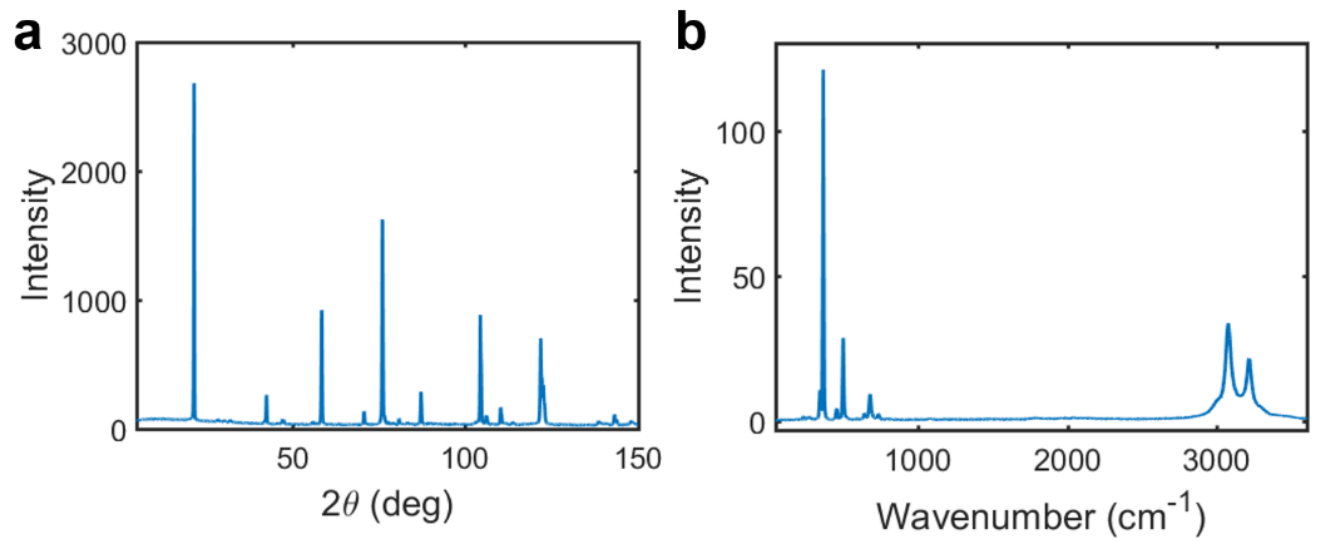

Figure S2. (a) X-ray diffraction and (b) Raman spectroscopy data confirm the crystallographic and chemical nature of boehmite. 


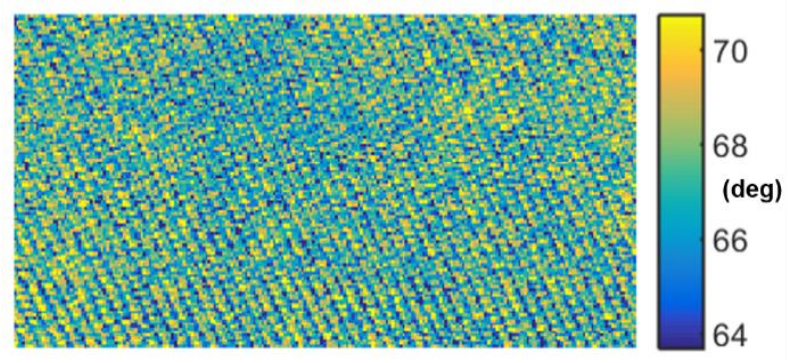

Figure S3. Lattice-resolution AFM phase image of boehmite (010) surface.
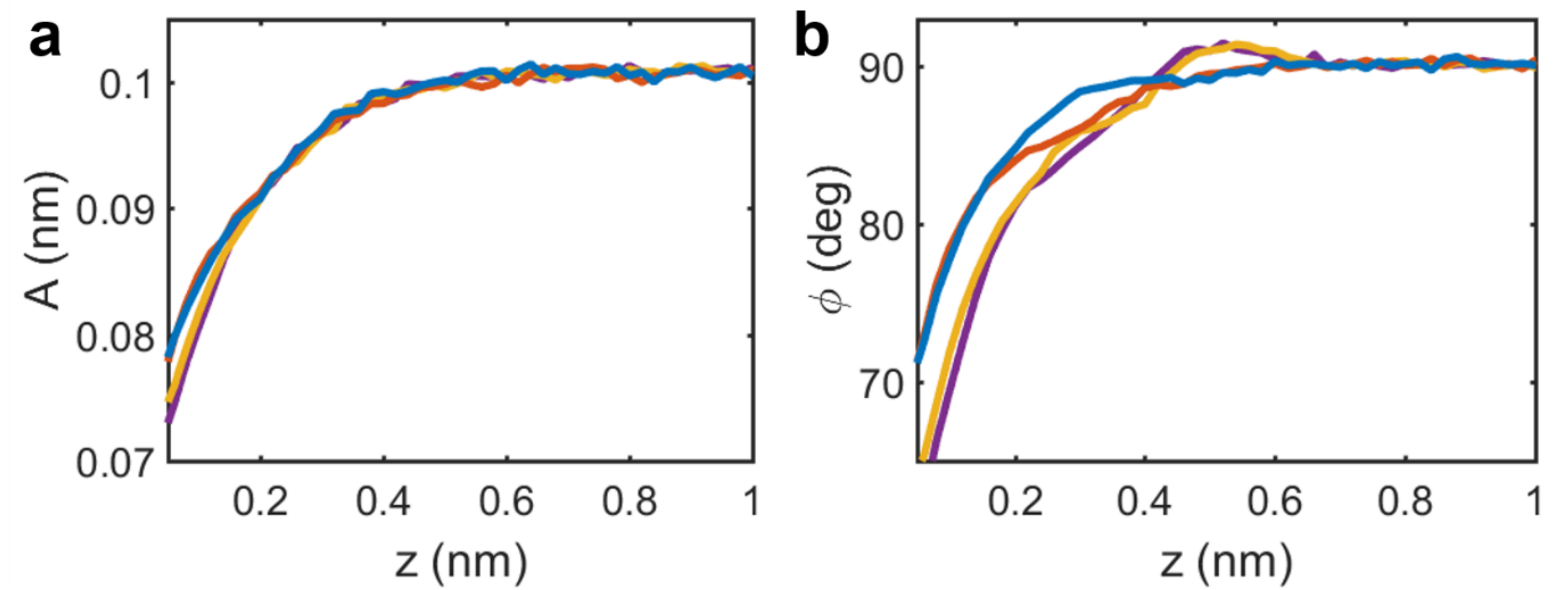

Figure S4. Example force curves in terms of (a) amplitude and (b) phase shift data. Oscillatory features are obvious in the phase shift data. 

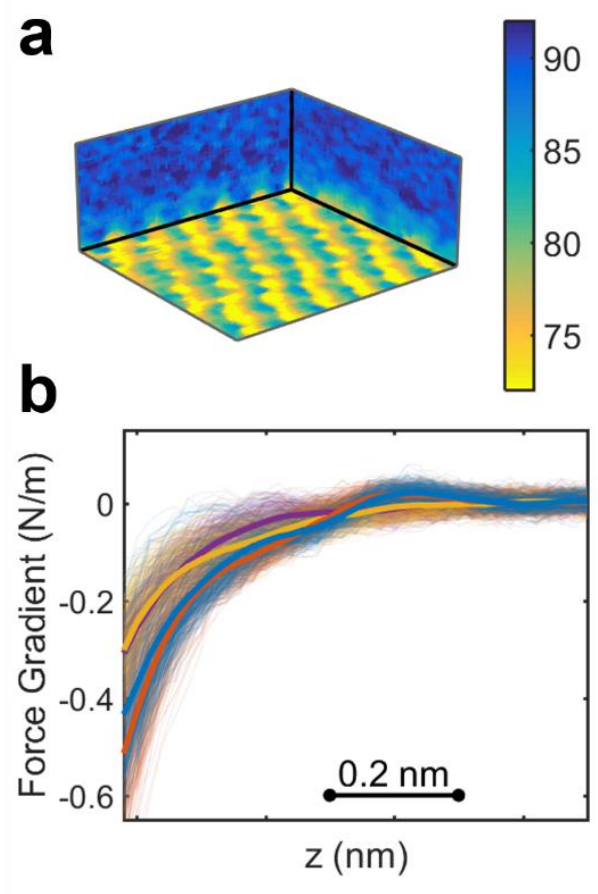

Figure S5. 3D data set of boehmite under similar conditions, using a different AC55 tip.

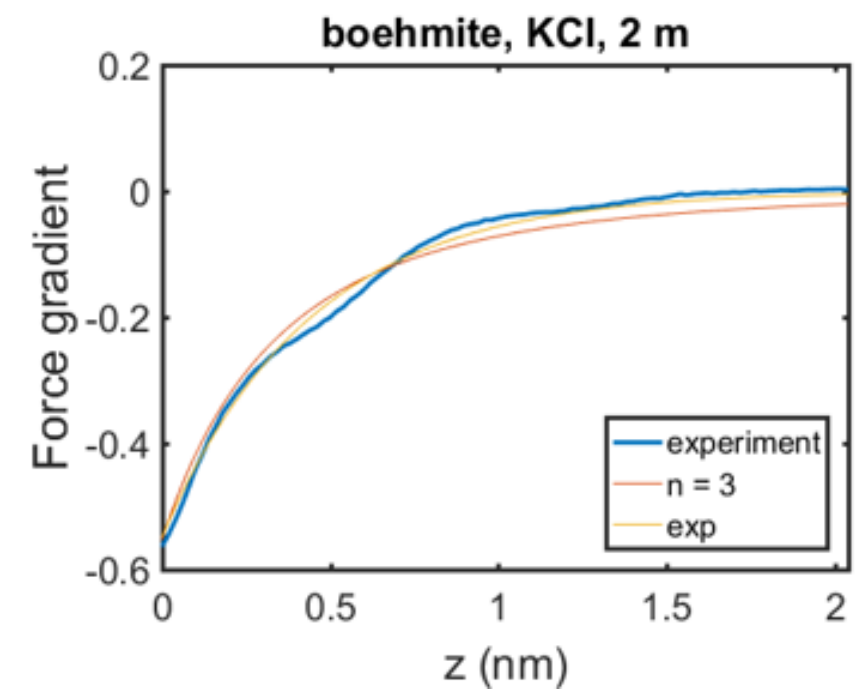

Figure S6. Long-range repulsive force persists at high ionic strength. 

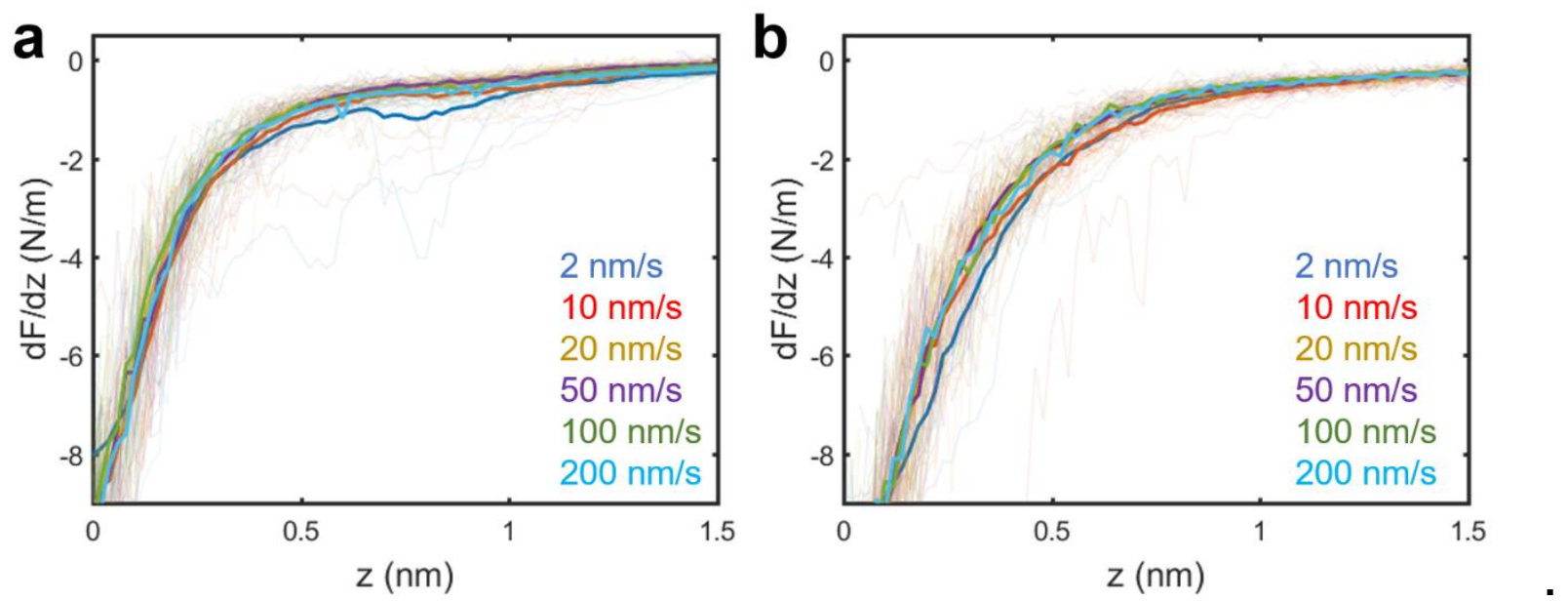

Figure S7. Force curves obtained for mica in $\mathrm{MgCl}_{2} 10 \mathrm{mM}$ solution with AC55 tip show that long-range interaction is not dependent on the vertical tip velocity.

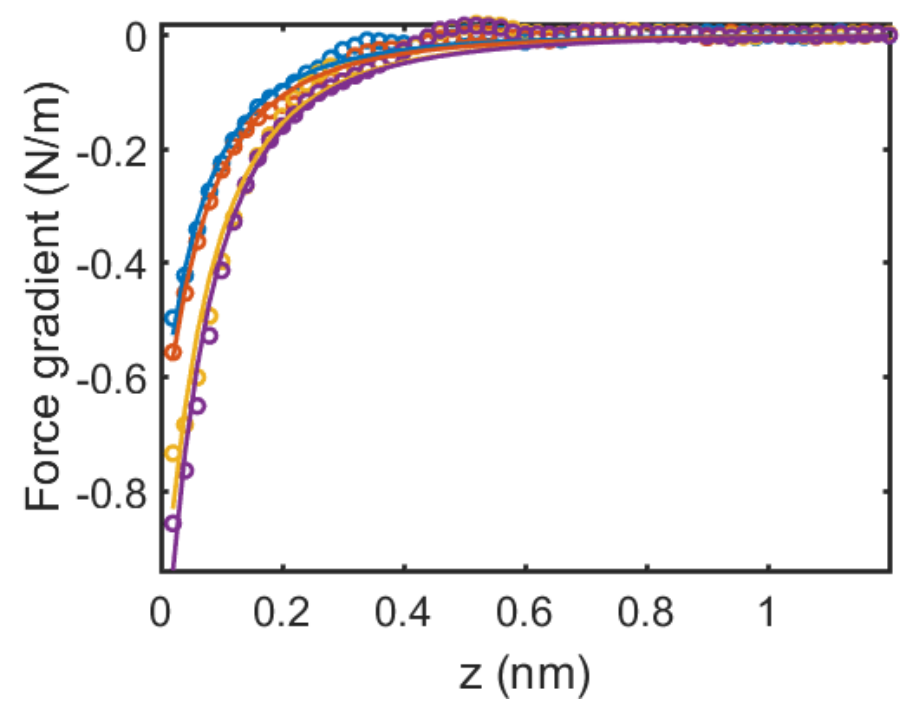

Figure S8. Examples of force curve fits to Equation 2 with $n=3$. 


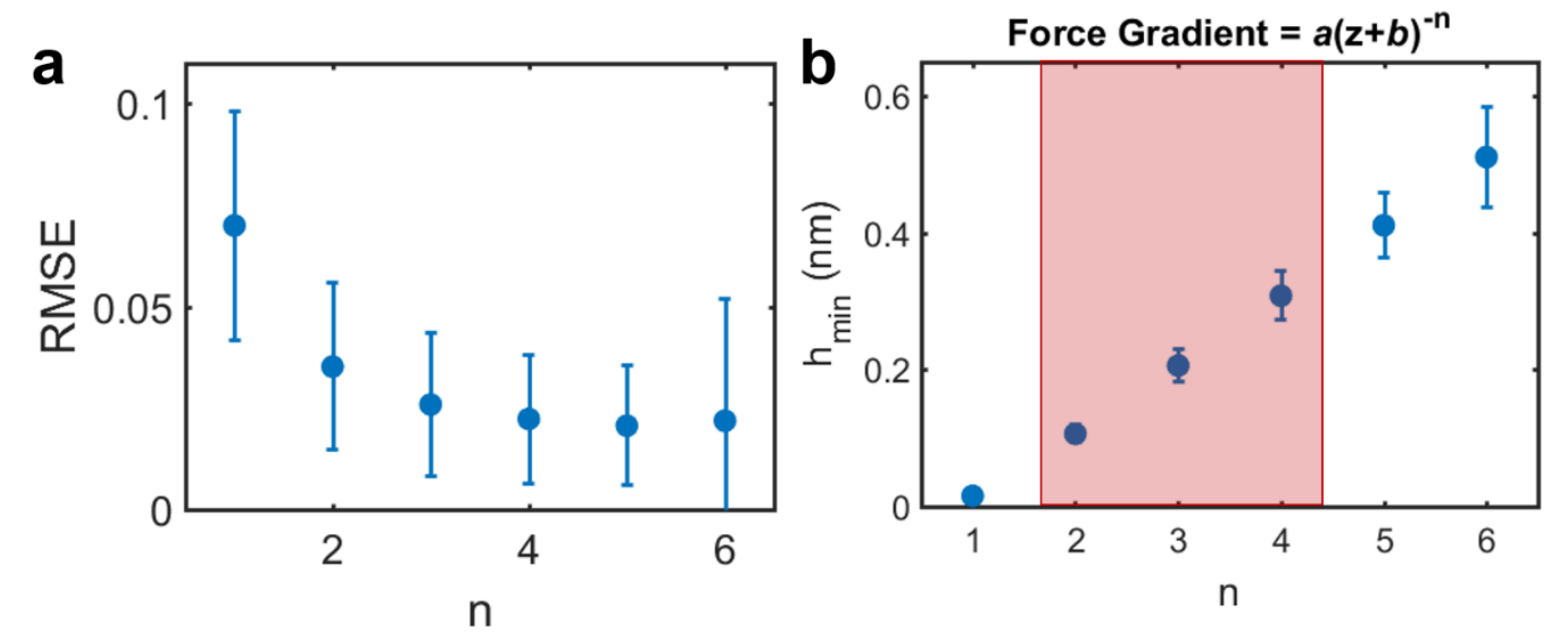

Figure S9. (a) Residual values for fits to Equation 2 with different values of $n$. (b) Equivalent height data as a function of $n$, obtained through the fitting parameter $b$.

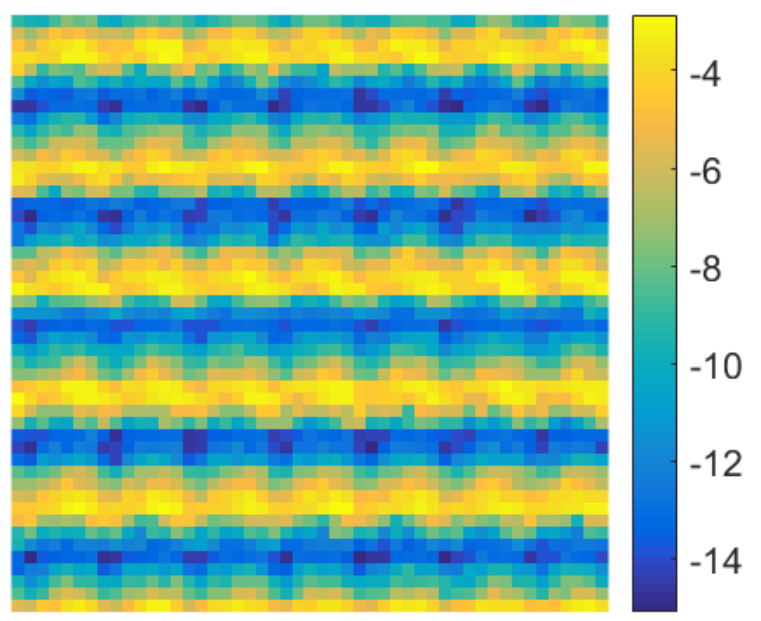

$\times 10^{-3}$

Figure S10. Lattice-scale patterning of the fitting parameter a for force curve fits to Equation 2 with $n=3$. 
a

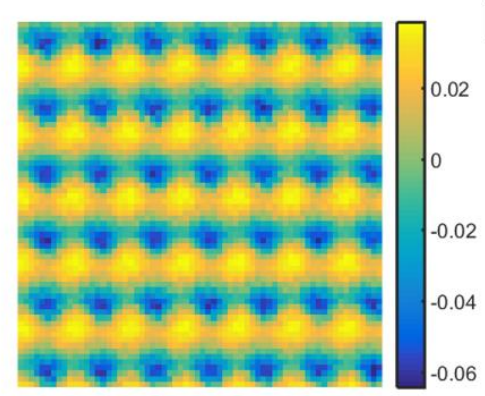

b

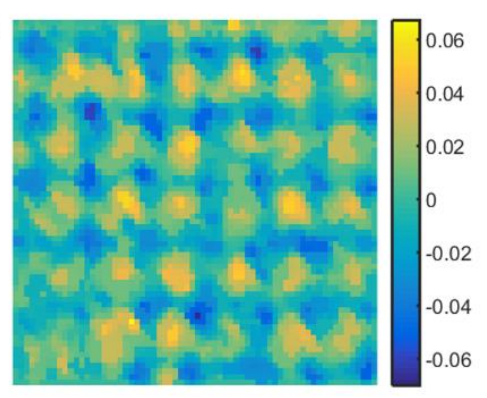

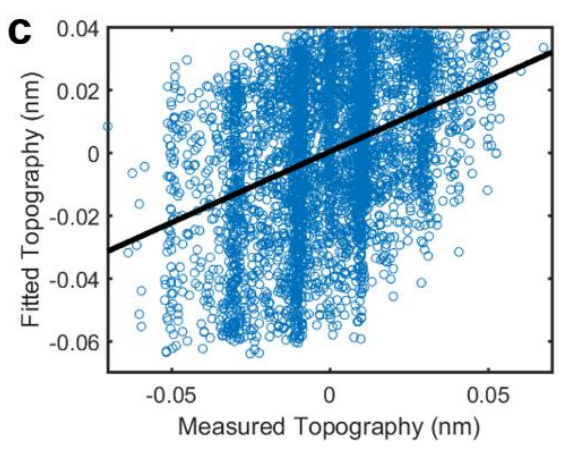

Figure S11. Surface topography (a) calculated from fits to Equation 2 with $n=3$ and (b) measured experimentally show (c) decent correlation.
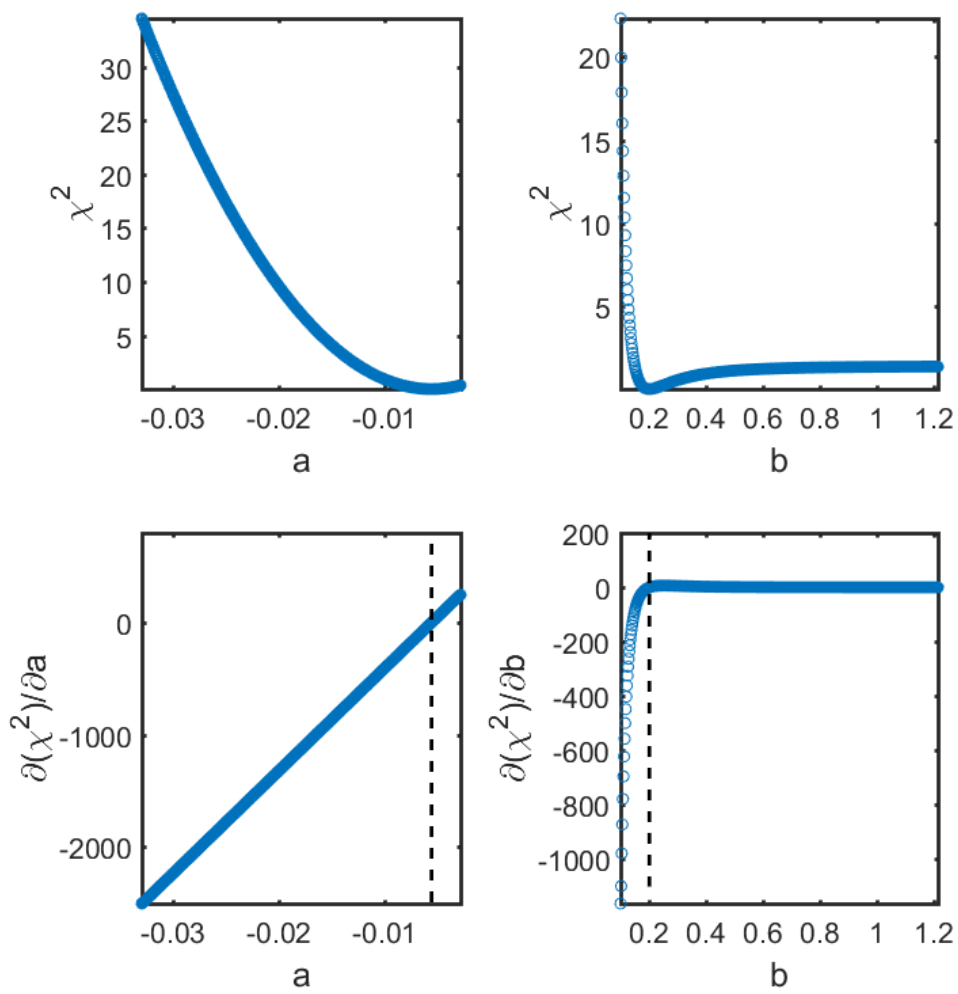

Figure S12. Calculation of the goodness-of-fit $\left(x^{2}\right)$ as a and b were varied from their optimal values. 

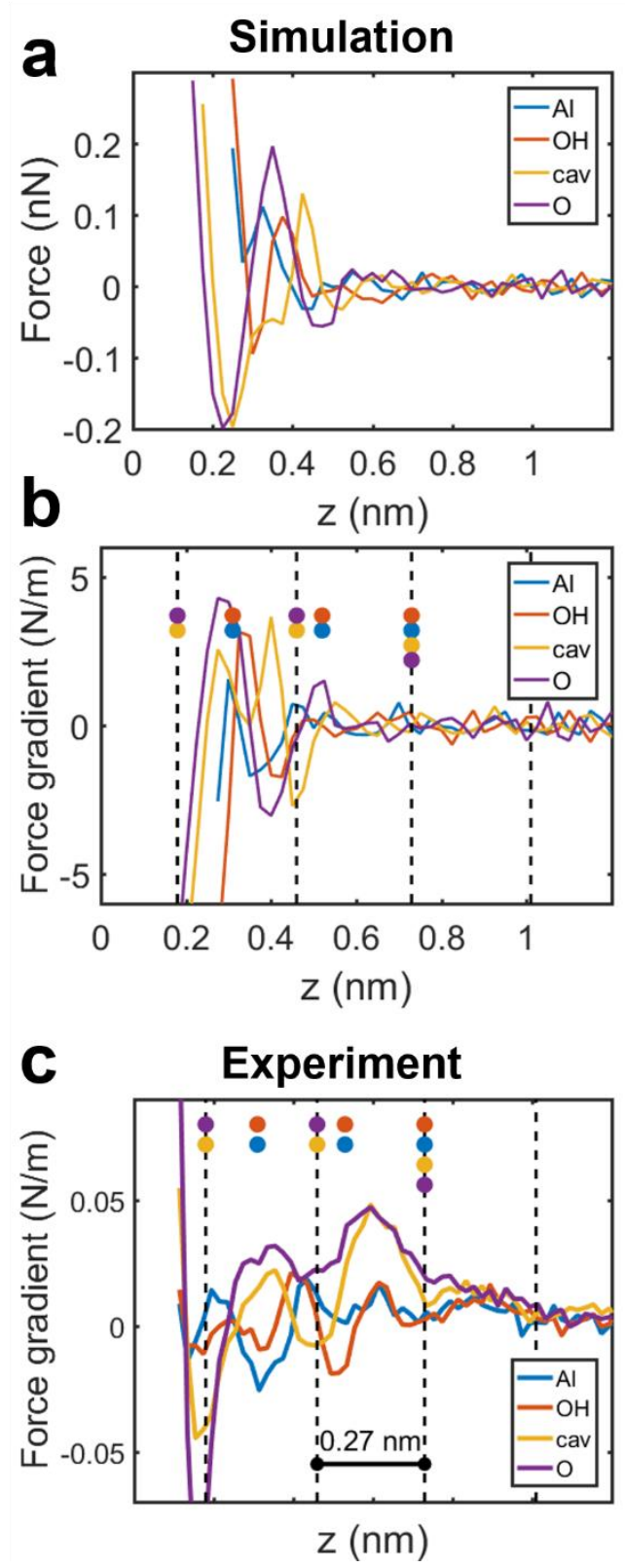

Figure S13. Conversion of energy landscape from solvent tip approximation to (a) force and (b) force gradient. (c) Direct comparison with experimental data. 

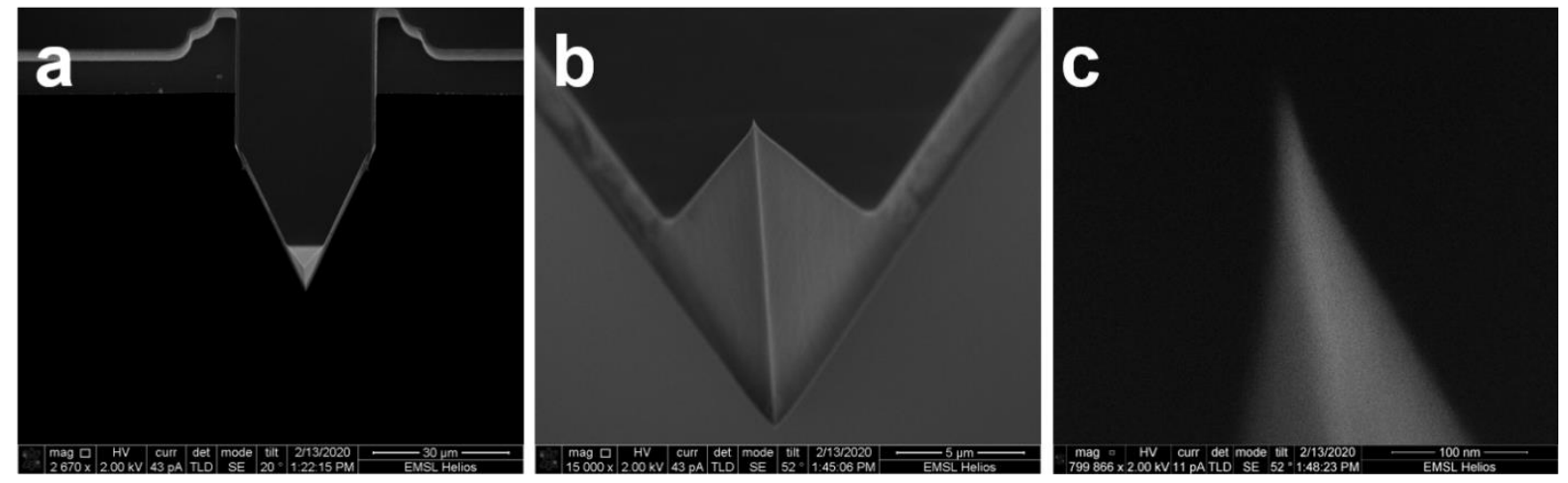

Figure S14. Scanning electron microscopy images of AFM tip.

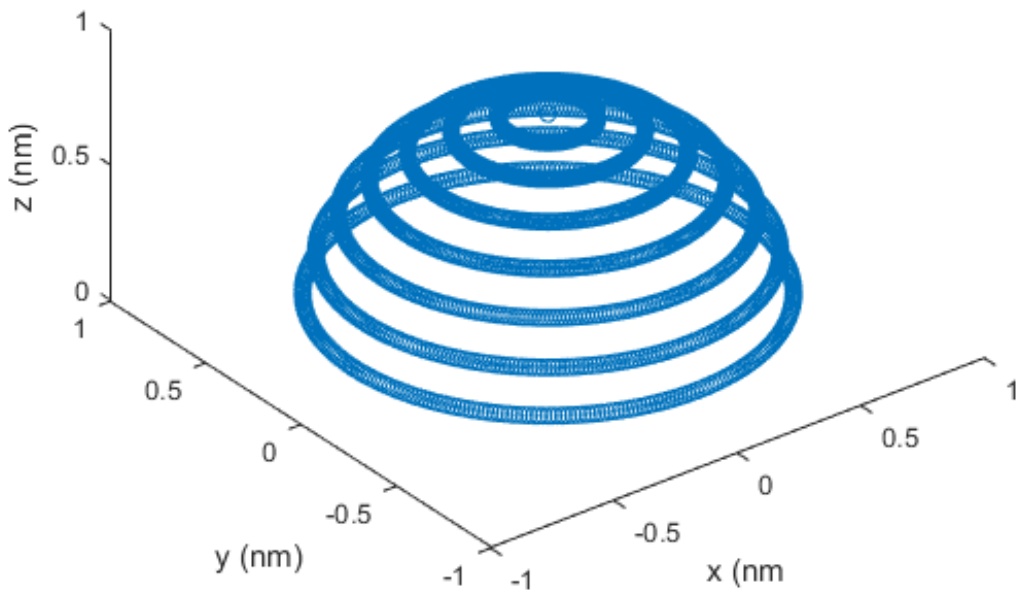

Figure S15. To simulate the effect of tip radius, the water density profiles are sampled and averaged from hemi-spherical surface; an approach that goes beyond the STA model. 

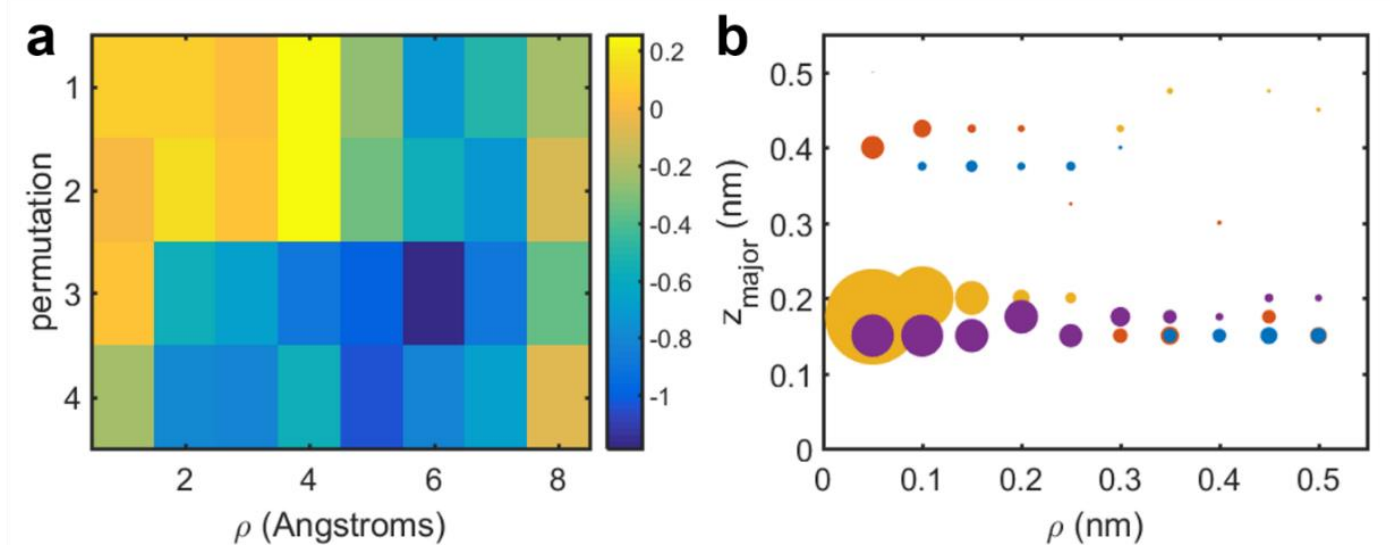

Figure S16. (a) Correlation between experimental and simulated energy landscape for tips of different radii rho. The data do not show particularly high correlation for any tip size, with smaller tip sizes showing more positive correlations. (b) Position and depth of first energy minima above specific lattice sites. The data shows that the relative order and magnitude of the energy minima change drastically as a function of tip size.

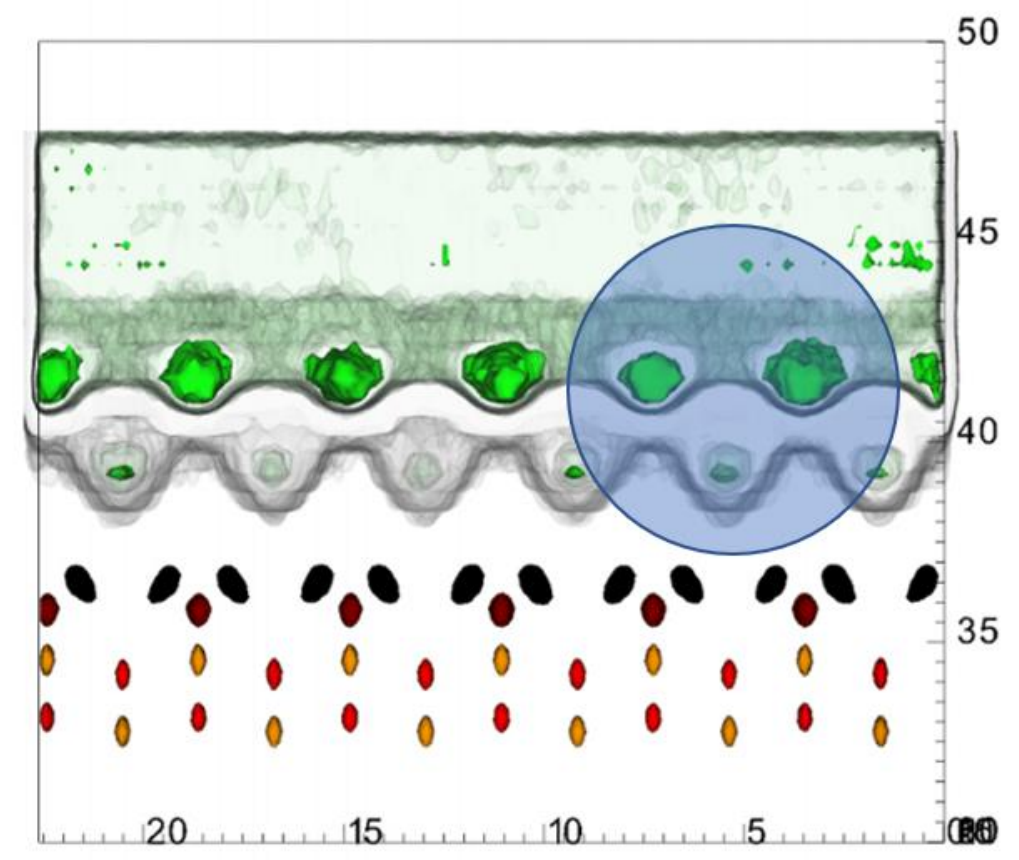

Figure S17. Snapshot of free energy landscape obtained from metadynamics simulation of boehmite-water-Lennard Jones sphere interactions. Dark red and black spots denote positions of surface hydroxyl oxygens and hydrogens, respectively. Green spots represent energy minima, while the blue circle demonstrates the size of the LJ sphere. 


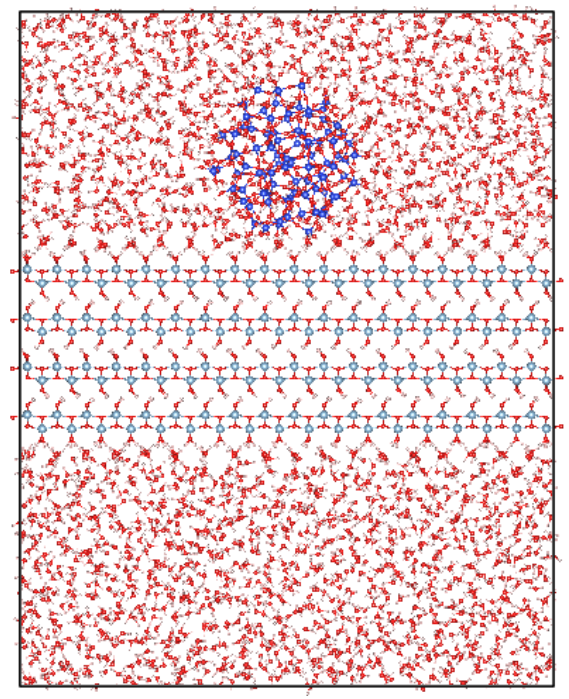

Figure S18. Snapshot of MD simulation of the boehmite-water-silica system.
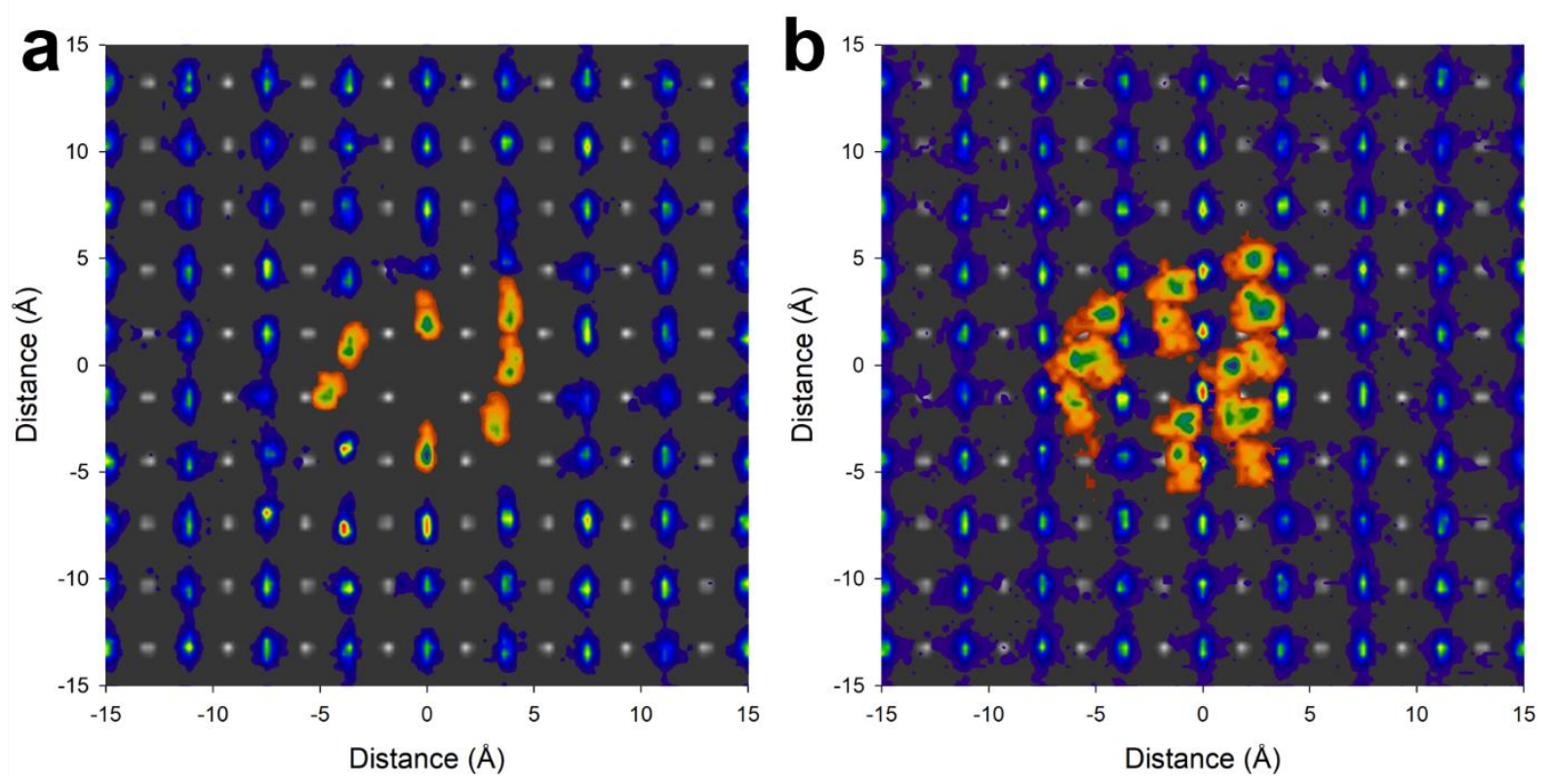

Figure S19. MD simulation of boehmite-water-silica system. Gray, blue, and orange/green spots denote hydroxyl oxygens, water molecules, and silanol groups, respectively. (a) At first free energy minimum, the hydroxyl groups on the surface of the silica particle are positioned in the first adsorbed water layer and interact directly through hydrogen bonding with the hydroxyl groups at the boehmite surface. (b) At the 2nd free energy minimum, the NP's hydroxyl groups interact directly through hydrogen bonding with the water molecules in the first adsorbed water layer 


\section{Movie Figure captions}

Movie 1. Space-averaged and background-subtracted 3D FFM data of boehmite-water interface in $\mathrm{pH} 11$ solution.

Movie 2. 3D FFM data of slices normal to the surface. Successive frames after displacement along [001] direction.

Movie 3. 3D FFM data of slices normal to the surface. Successive frames after displacement along [100] direction. 


\section{References}

1. W. Smith, T. R. Forester, DL_POLY_2. 0: A general-purpose parallel molecular dynamics simulation package. J Mol Graphics 14, 136-141 (1996).

2. R. T. Cygan, J. J. Liang, A. G. Kalinichev, Molecular Models of Hydroxide, Oxyhydroxide, and Clay Phases and the Development of a General Force Field. $J$ Phys Chem B 108, 1255-1266 (2004).

3. O. Teleman, B. Jönsson, S. Engström, A molecular dynamics simulation of a water model with intramolecular degrees of freedom. Mol Phys 60, 193-203 (1986).

4. W. G. Hoover, Canonical dynamics: Equilibrium phase-space distributions. Phys Rev $A$ 31, 1695 (1985).

5. P. P. Ewald, Die Berechnung Optischer Und Elektrostatischer Gitterpotentiale. Ann Phys 369, 253-287 (1921).

6. X. Zhang et al., Direction-specific interaction forces underlying zinc oxide crystal growth by oriented attachment. Nat Commun 8, 835 (2017).

7. S. N. Kerisit, J. J. De Yoreo, Effect of Hydrophilicity and Interfacial Water Structure on Particle Attachment. The Journal of Physical Chemistry C 124, 5480-5488 (2020).

8. A. Ben-Naim, Molecular Theory of Solutions. (Oxford University Press, Oxford, 2006).

9. S. Plimpton, Fast Parallel Algorithms for Short-Range Molecular-Dynamics. J Comput Phys 117, 1-19 (1995).

10. A. N. Christensen, M. S. Lehmann, P. Convert, Deuteration of crystalline hydroxides. Hydrogen bonds of g-AlOO (H, D) and g-FeOO (H, D). Acta Chem Scan 36, 303-308 (1982).

11. M. Levitt, M. Hirshberg, R. Sharon, K. E. Laidig, V. Daggett, Calibration and testing of a water model for simulation of the molecular dynamics of proteins and nucleic acids in solution. J Phys Chem B 101, 5051-5061 (1997).

12. A. Laio, M. Parrinello, Escaping free-energy minima. Proc Natl Acad Sci U S A 99, 12562-12566 (2002).

13. G. A. Tribello, M. Bonomi, D. Branduardi, C. Camilloni, G. Bussi, PLUMED 2: New feathers for an old bird. Comp Phys Commun 185, 604-613 (2014).

14. P. Raiteri, A. Laio, F. L. Gervasio, C. Micheletti, M. Parrinello, Efficient reconstruction of complex free energy landscapes by multiple walkers metadynamics. J Phys Chem $B$ 110, 3533-3539 (2006).

15. D. Argyris, A. Phan, A. Striolo, P. D. Ashby, Hydration Structure at the $\alpha-\mathrm{Al}_{2} \mathrm{O}_{3}(0001)$ Surface: Insights from Experimental Atomic Force Spectroscopic Data and Atomistic Molecular Dynamics Simulations. J. Phys. Chem. C 117, 10433-10444 (2013). 